\title{
Interaction of Rupture Zones of Adjacent Anchor Plates in an Analogical Medium
}

\author{
Hichem Abbad \\ Djillali Liabes university \\ Dept. Civil Engineering, \\ Fac.Technology Labo. Civil \\ Engineering \& Environment - \\ LGCE \\ Sidi Bel Abbes, Algeria \\ hi_abbad@yahoo.fr
}

\author{
Mourad Meghachou \\ Djillali Liabes University \\ Dept. Civil Engineering, \\ Fac. Technology Labo. \\ Civil Engineering \& \\ Environment -LGCE \\ Sidi Bel Abbes, Algeria \\ mourad_meghachou@univ- \\ sba.dz
}

\author{
Chahinez Dekar \\ Djillali Liabes University \\ Dept. Civil Engineering, \\ Fac.Technology Labo. \\ Civil Engineering \& \\ Environment -LGCE \\ Sidi Bel Abbes, Algeria \\ ch_gecicat@yahoo.fr
}

\author{
Sidi Mohamed Daoud \\ Djillali Liabes University \\ Dept. Civil Engineering, \\ Fac. Technology Labo. \\ Civil Engineering \& \\ Environment -LGCE \\ Sidi Bel Abbes, Algeria \\ daoudmed22@yahoo.fr
}

\begin{abstract}
In this paper, an experimental study which required the design and implementation of a model containing plastic granules powder to simulate a natural environment, is presented. The latter is subjected to the removal of anchor plates. For each test, several digital photographs are taken to materialize different deformed configurations during the pullout process. These photos processed in couples by the 7D software (image correlation) giving the evolution of the displacement field and plane strain analogical environment. Particular attention is paid to the discussion of the interference of rupture zones of neighboring anchors by reducing the axis between plates.
\end{abstract}

Keywords- correlation of images; small-scale model; anchor plates; granular medium; shear; plane problem.

\section{INTRODUCTION}

The anchor plates are light structural elements employed to withstand uplift forces as experienced by transmission towers, masts and structures subject to buoyancy effects as offshore stations. Theoretical studies and experimental models of reduced contiguous plates embedded in granular soil [1-5] showed the influence of soil anchor, of the geometry of the plates, of the depth of the anchor and of the distance between two adjacent plates in their behavior.

What actually happens in the environment of two adjacent anchor plates however, is always instructive. Kinematic analysis, through physical modeling, allows the investigation of actual behavior. For example, ask the following question: what happens in a medium surrounding two contiguous anchor plates positioned at different distances from each other? It would be interesting to analyze the interaction of the deformed plates around these areas. In this paper, we present an experimental study that describes the displacement field and the strain field in a medium subjected to the removal of two adjacent plates, answering the first question. The use of an analogical environment in this kind of modeling provides an approach to the understanding of the phenomenology of real soils, as both materials have certain properties in common such as incompressibility grain and rubbing character.

\section{EXPERIMENTAL MODEL}

The model on which we have carried out the tests, is a rectangular device of $100 \times 80 \times 5 \mathrm{~cm}$ dimension, designed to contain plastic granules (analogical material) simulating a granular medium. The front wall of the model is made of a glass plate $6 \mathrm{~mm}$ thick, with a constant refractive index throughout its surface. The other walls are formed by plates rigid with wood. Wooden smoothed parallelepipeds of $5 \times 5 \times 2$ $\mathrm{cm}$ dimensions model the anchor plates. They are in contact with threaded rods of $50 \mathrm{~cm}$ in length. Nuts are welded to an attachment to the chassis of the model, and the tearing of the two plates can be controlled by unscrewing the rods. A strictly vertical movement is imposed on the anchor plates as they are guided by $5 \mathrm{~mm}$ thick rails, mounted on the rear wall, preventing any rotation, as shown in Figure 1.

The plastic grains average diameter is $1 \mathrm{~mm}$, different colors (red, blue, yellow, green and black) are mixed to materialize a visually contrasting environment (natural speckle). To obtain a compact and homogeneous medium, the material is deposited uniformly over the entire width of the tray by small $2 \mathrm{~cm}$ thick layers. Each layer is compacted through a metallic rammer compaction equal to $2.5 \mathrm{~kg}$ mass by exercising 75 blows with a drop height of $30 \mathrm{~cm}$. The medium has a void index of 0.321 , corresponding to a relative density of $\mathrm{D}_{\mathrm{r}}=96 \%\left(\mathrm{e}_{\min }=0.302, \mathrm{e}_{\max }=0.855\right)$, the unit weight of the dry analogical material is $\gamma_{\mathrm{d}}=14,6 \mathrm{kN} / \mathrm{m}^{3}$, its cohesion is null and its natural friction angle it $\varphi=39^{\circ}$. In view of the very low stresses involved, plastic grains constituting the studied areas are considered indeformable.

To describe the displacement field and strain over the anchor plates, several series of tests were conducted. For each test performed, a series of digital photos, with a resolution of $3200 \times 2400$ pixels, is taken from the front and with the axis of the shooting perpendicular to the plane of the model. These photos materialize different deformations in the granular medium, caused by the tearing of the anchor plates. The recorded images are further processed to determine the strain field using the 7D software. It is a method of correlation between two images [6]: they are compared using the correlation coefficient of the gray level. In other words, the 
points of the original image are found on successive images with a measurement uncertainty of about 0.1 pixel, or 0.01 pixel in small deformation. The image for which the strain is analyzed is divided into a number of square grid elements of 20x20 pixels.

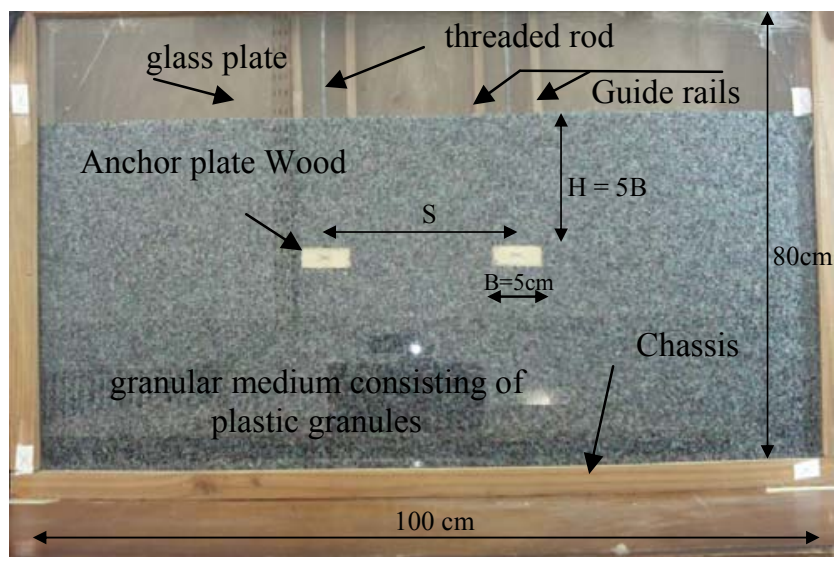

Fig. 1. View of the model

\section{DISPLACEMENTS AND DEFORMATIONS OVER THE ANCHOR PLATES}

Highlighting the influence of the distance between the centers of adjacent anchor plates on the behavior of the surrounding granular medium is deliberately kinematics, ignoring any measurement of the stress state. The tests in question were conducted for anchored at a depth of $\mathrm{H}=5 \mathrm{~B}$, where $B$ is the width of the plates. The spacing axes distance $S$ varied as follows: $S=3 B, S=4 B, S=5 B$ and $S=7 B$. During image processing, a field of study is defined by the operator. We will follow in the evolution of displacements and deformations in this area. The tearing or lifting imposed on the anchor plates, occurs at constant pitches in the order of $0.266 \mathrm{~mm}$ that correspond to 1 pixel.

\section{A. Displacement field}

Figure 2 reproduces graphically the trajectories of grains for a tearing of 20 pixels and for a distance between the axes of respective anchor plates of $3 \mathrm{~B}, 4 \mathrm{~B}, 5 \mathrm{~B}$ and $7 \mathrm{~B}$. Note that the grain movement is growing vertically along the axis of the plate, and horizontally to the edges of it. The displacement vectors are plotted with an amplification coefficient of 7 $(700 \%)$. First, we find that the displacement vectors are generally vertical and oriented upwards, the trajectories of grains damp, almost, approaching a horizontal plane parallel to the free surface of the massif, following an upwardly curved arc.
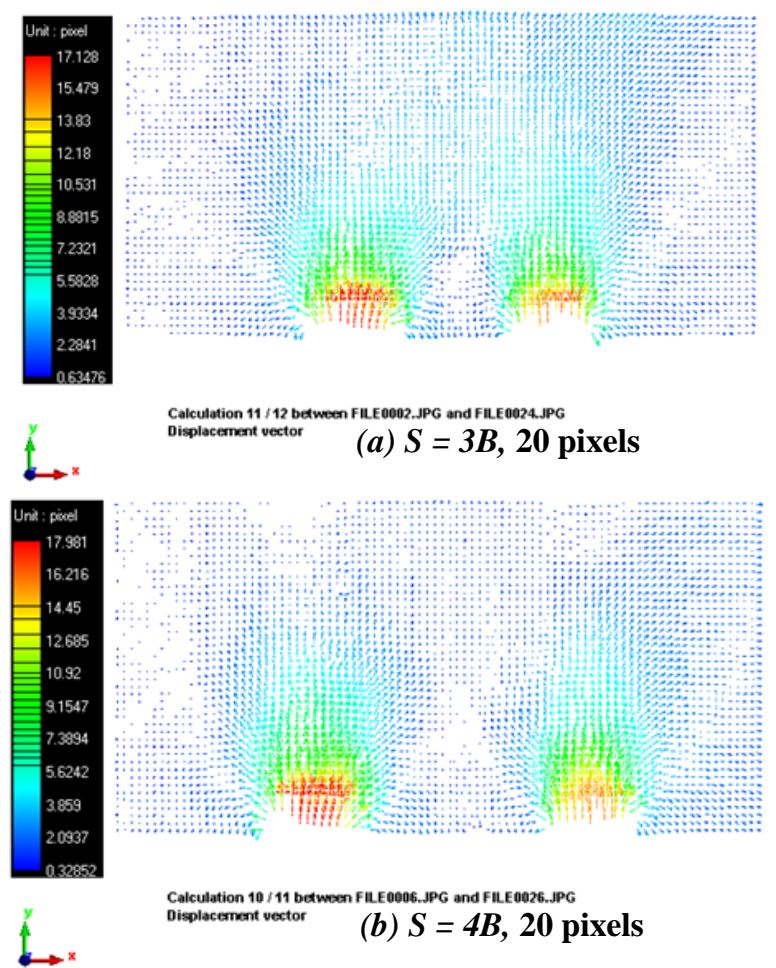

(b) $S=4 B, 20$ pixels
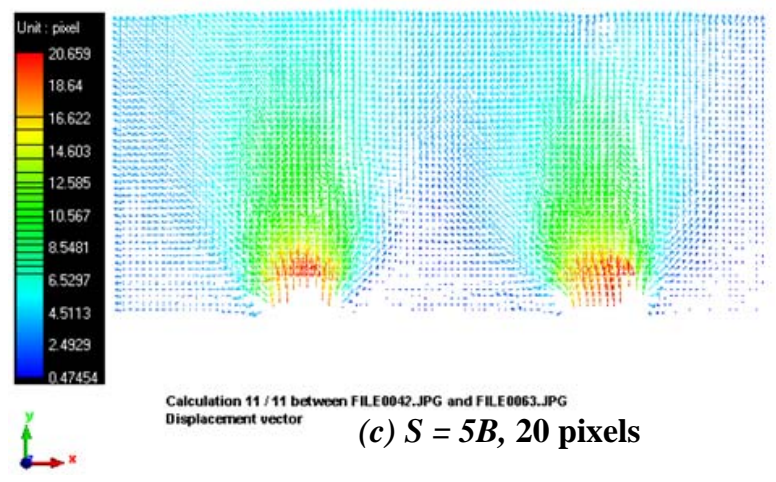

(c) $S=5 B, 20$ pixels
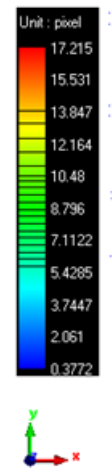

Calculation 11 / 12 between FLLE0002.JPG and FILE0023.JPG

(d) $S=7 B, 20$ pixels

Fig. 2. Displacement vectors for a pullout of 20 pixels and for different distances between axes of plates. 
Figure 3 represents the vertical displacement fields of the material applied by two anchorage plates with a space between axes respectively of $3 \mathrm{~B}, 4 \mathrm{~B}, 5 \mathrm{~B}$ and $7 \mathrm{~B}$. Tear is 20 pixels. Locally over each plate, the medium behaves in a manner similar to an isolated plate. Note that the formation of a "rigid wedge" over each plate confirms the high compactness of the material. According to the tear's intensity of the plates, a large displacement gradient is set up on a slightly curved triangular outline confirming the existence of the rigid wedge above the plates.

We note that beyond the distance of $S=7 B$, plates act independently on the material. At the distance of $S=3 B$, the interaction of rupture zones of each plate creates a bulb of vertical displacement which reaches the value of 2.76 pixels at the surface of the massif at midway between the axes of the plates. The bulge of the grains is limited by inclined lines passing by the edges of the plates. The inclination of these lines is about $41^{\circ}$ relative to the vertical. This inclination is substantially equal to the internal angle of friction of an analogical soil $\left(\varphi=39^{\circ}\right)$. These lines materialized slip lines (rupture surfaces). This mode of rupture has been observed by several researchers who have studied the phenomenon of interaction of anchor plates in granular soils $[8,9]$.

\section{B. Deformation field (maximum shear)}

Figure 4 reproduces the strain fields (maximum shear) in the granular medium for a plate uplift of 20 pixels and for a spacing between axes of $3 \mathrm{~B}, 4 \mathrm{~B}, 5 \mathrm{~B}$ and $7 \mathrm{~B}$. It is noticed that the shear (bulb shear) at the surface of the massif, midway the plates axes is larger when the plates move towards each other. The shear intensity reaches the value of $1.02 \%$ for a spacing of $3 \mathrm{~B}$ whereas it does not exceed $0.23 \%$ for a spacing of $5 \mathrm{~B}$ and $0.12 \%$ for a spacing of $7 \mathrm{~B}$. Note the edge effect at the corners of bases of the upper faces of the plates.

\section{CONCLUSION}

The experimental study we conducted was based mainly on the evolution of the granular medium subjected to the pullout of anchor plates. The pullout tests performed were used to quantify the vertical displacement field and the deformations of granular medium over "anchor plates" with the image correlation technique. The choice of a pullout of 20 pixels (imposed displacement) is justified by the need to present strain fields corresponding to a state of failure. A multitude of coupled photos were processed, up to a pullout of 35 pixels and more. Beyond 18 pixels, the deformed configuration is virtually unchanged, which shows that at this stage of sollicitation, the medium has reached a state of rupture and plasticity state occurs. The interaction of rupture zones of neighboring plates anchored at a depth of 5B is studied kinetically by varying the distance between the axes of the plates. It appears that a minimum distance between axes of approximately seven times the width of the plate (7B) is needed for the two adjacent anchoring plates to act independently on the medium. The formation of a "rigid wedge" on the top of each plate was demonstrated. The failure mode observed during the pullout process is in agreement with the previous results.
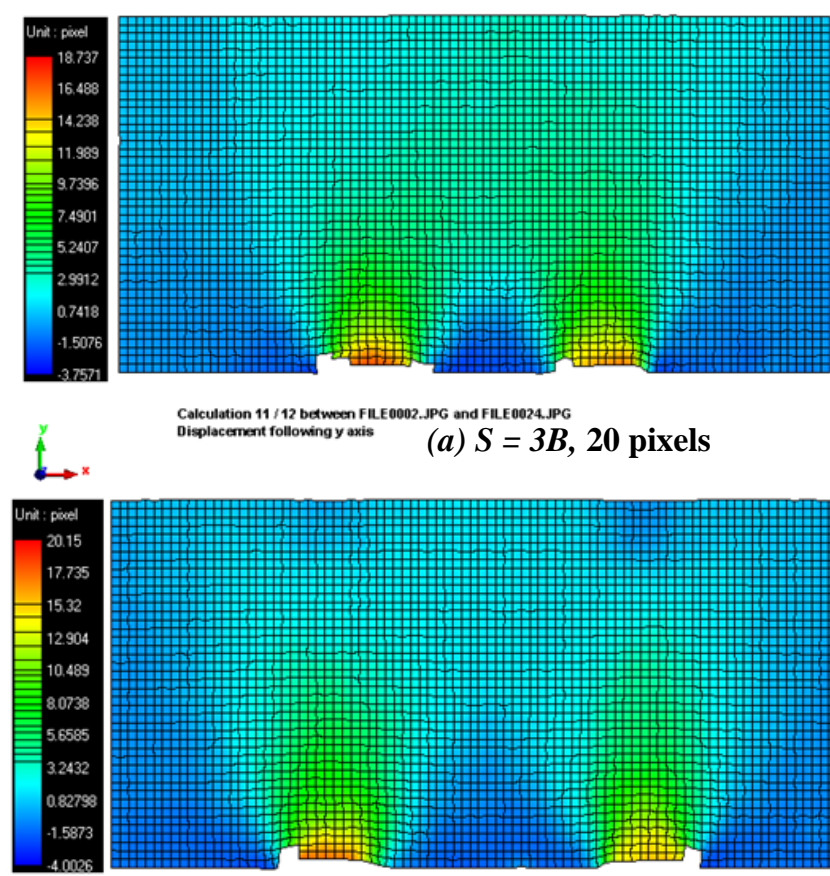

Calculation $11 / 12$ between FLLE0002.JPG and FILE0024.JPG

(a) $S=3 B, 20$ pixels
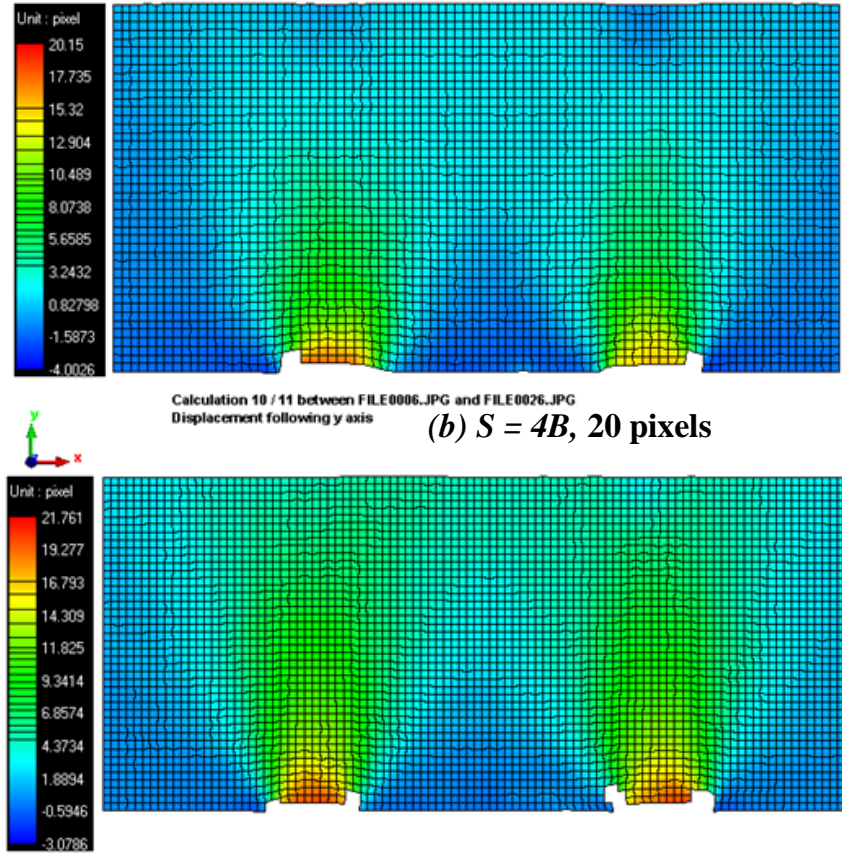

Calculation 10/11 between FLLE0006.JPG and FLLE0026.JPG
Displacernent following y axis
(b) $S=4 B, 20$ pixels

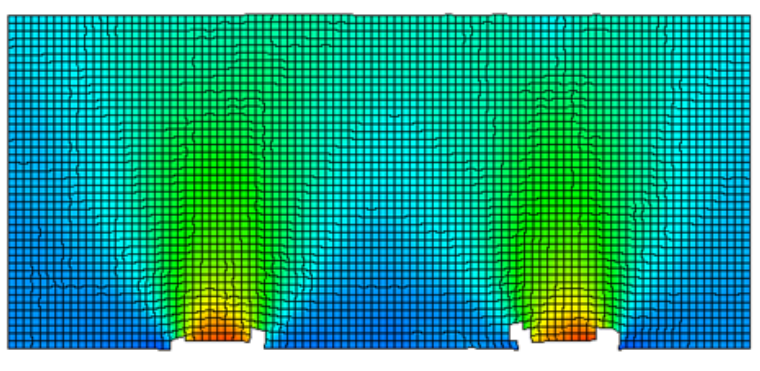

$t_{x \rightarrow x}$

Calculation $11 / 11$ between FLLE0042.JPG and FILE0063.JPG

(c) $S=5 B, 20$ pixels
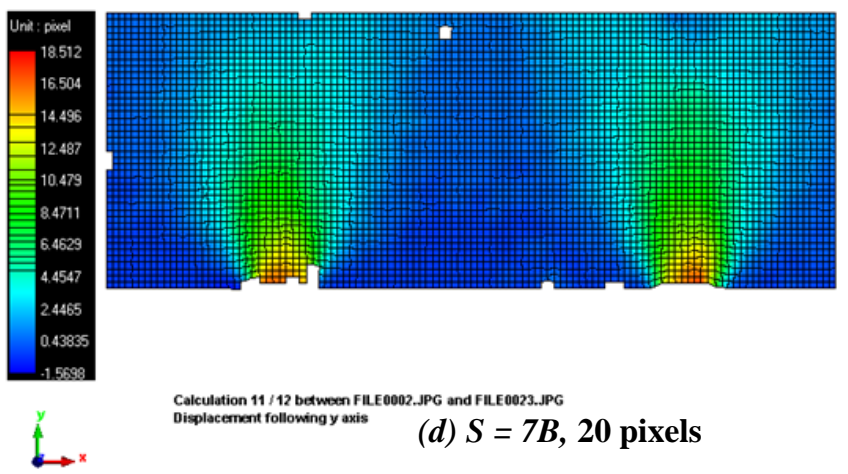

Calculation $11 / 12$ between FLLE0002.JPG and FLLE0023.JPG
Displacenent following y axis

(d) $S=7 B, 20$ pixels

Fig. 3. Vertical displacement field for a pullout of 20 pixels and for different distances between axes of plates. 

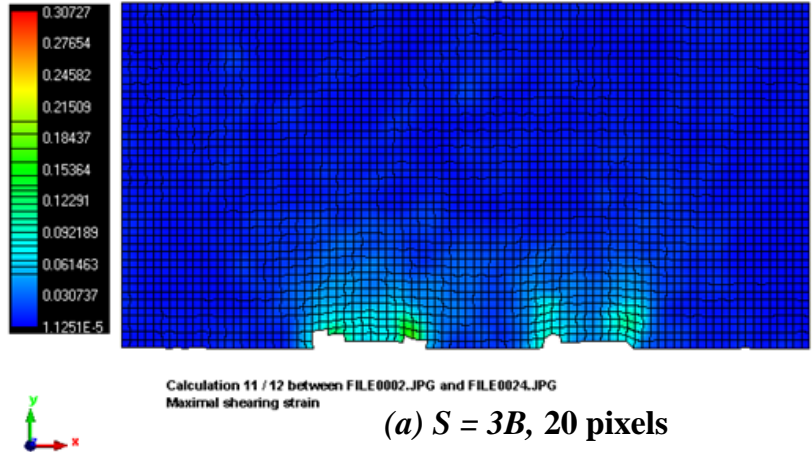

Calculation $11 / 12$ betwo

(a) $S=3 B$, 20 pixels
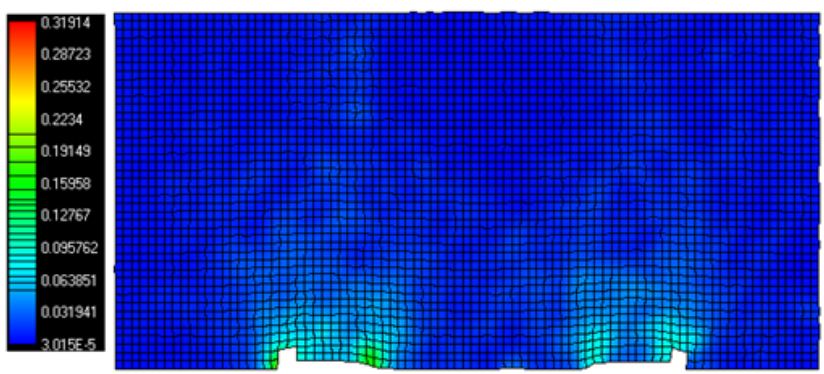

Calculation $10 / 11$ between FILE0006.JPG and FLEE0026.JPG
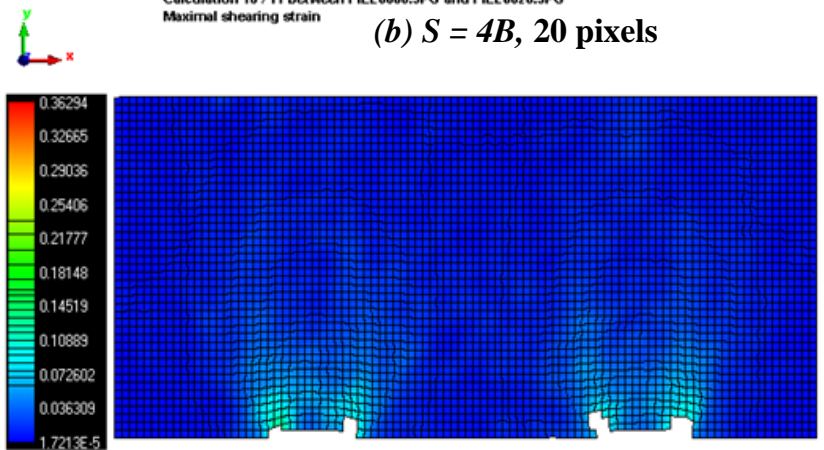

Calculation 11 / 11 between FlLE0042.JPG and FILE0063.JPG

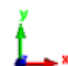

Moximal ș

(c) $S=5 B, 20$ pixels
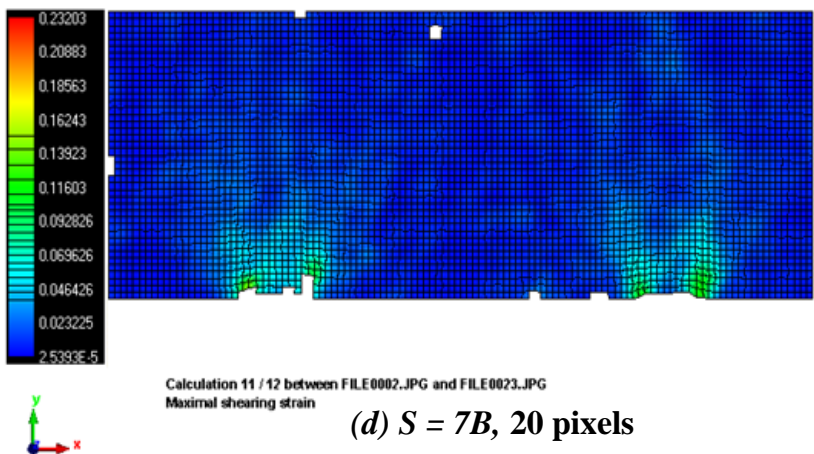

Calculation $11 / 12$ between FILE0002.JPG and FLEF0023.JPG

(d) $S=7 B, 20$ pixels

Fig. 4. Deformation field (maximum shear) for a pullout of 20 pixels and different distances between axes of plates.

\section{REFERENCES}

[1] G. G. Meyerhof, J. I. ADAMS, "The ultimate uplift capacity of foundations", Canadian Geotechnical Journal, Vol. 5, No. 4, pp. 225244, 1968

[2] E. J. Murray, J. D. Geddes, "Uplift of anchor plates in sand“, Journal of Geotechnical Engineering, Vol. 113, No. 3, pp. 202-215, 1987

[3] T. H. Hanna, R. Sparks, M. Yilmaz, "Anchor behavior in sand“, Journal of the Soil Mechanics and Foundations Division, Vol. 98, No. 11, pp. 1187-1208, 1972

[4] J. D. Geddes, E. J. Murray, "Plate anchor groups pulled vertically in sand“, Journal of Geotechnical Engineering, Vol. 122, No. 7, pp. 509524, 1996

[5] K. Kouzer, J. Kumar, "Vertical uplift capacity of equally spaced horizontal strip anchors in sand", International Journal of Geomechanics, Vol.9, No. 5, pp. 230-236, 2009.

[6] P. Vacher, S. Dumoulin, F. Morestin, S. Mguil-Touchal, "Bidimensional strain measurement using digital images", Proceedings of the Institution of Mechanical Engineers, Part C: Journal of Mechanical Engineering Science, Vol. 213, No. 8, pp. 811-817, 1999

[7] H. Abbad, M. Meghachou, P. Vacher, "Interaction of subjacent zones due to contiguous shallow foundations in analogical medium", European Journal of Environmental and Civil Engineering, Vol.15,No. 3, pp. 391409, 2011.

[8] K. M. Kouzer, J. Kumar, "Vertical uplift capacity of two interfering horizontal anchors in sand using an upper bound limit analysis", Computers and Geotechnics, Vol. 36, No. 6, pp. 1084-1089, 2009

[9] J. Kumar, K. M. Kouzer, "Vertical uplift capacity of a group of shallow horizontal anchors in sand ", Géotechnique, Vol. 58, No. 10, pp. 821823,2008 